\title{
Arbeits- und Personalpolitik für die 2020er-Jahre - Ausgangslage und Meilensteine der vergangenen 20 Jahre
}

\author{
R. Overhage
}

\section{Einleitung: Die Strategie der Lebenszeitverlängerung.}

Bis in die 90er-Jahren und zum Teil noch darüber hinaus wurde europaweit ein vorzeitiger Ruhestand positiv bewertet. Ein wesentliches Argument: Arbeitsplätze für die junge Generation würden freigestellt. Ein weithin akzeptiertes Argument, denn die Arbeitslosigkeit war wesentlich höher als heute. Dieser Logik folgte auch die Altersteilzeit, indem die Forderung an die Einstellung der Nachwuchskräfte gekoppelt war. Der Vorruhestand wurde im Rahmen des EGKS (Europäische Gemeinschaft für Kohle und Stahl)-Vertrages auch finanziell unterstützt. Dadurch konnte ein Kapazitätsabbau sozialverträglich, ohne betriebsbedingte Kündigungen, gestaltet werden. Dieser war je zur Hälfte durch die Unternehmen der Montanindustrie umlagefinanziert und zur anderen Hälfte aus staatlichen $\mathrm{Zu}-$ schüssen gedeckt.

Der EGKS-Vertrag lief aus; die Europäischen Verträge folgten dann einer anderen Logik. Es ging um einen einheitlichen Binnenmarkt sowie mit dem Bolognaprozess um einheitliche Bildungsabschlüsse. In Deutschland versprach man sich dadurch auch eine Verkürzung der Studienzeiten. Die Einführung von G8 sollte zudem die Schulzeit verkürzen. Die schrittweise Anhebung des Renteneintritts war ein weiterer Baustein der Strategie der Lebensarbeitszeitverlängerung. Darüber hinaus wurde die kapitalgedeckte „Riesterrente“ mit einem staatlichen Zulagesystem als Ergänzung des umlagefinanzierten Rentensystems eingeführt.

\section{Demografiestrategie der Bundesregierung}

Die Politik der Lebensarbeitszeitverlängerung war nur eine Facette, den Herausforderungen des demografischen Wandels zu begegnen. Die Enquetekommissionen, die der Deutsche Bundestag zum Thema eingesetzt hatte, setzen das Thema „Demografie“ auf die Agenda und lieferten Informationen sowie Prognosen. Deutlich wurde, dass in Zukunft mit im 
Durchschnitt älteren Beschäftigten Produktion und Dienstleistungen erbracht werden müssen. Und es setzte sich die Überzeugung durch, dass dies eine gemeinsame Aufgabe von Staat, Wirtschaft und Gesellschaft ist. Die Bundesregierung gab daher 2012 den Impuls zur Demografiestrategie $^{1}$. Im Kern war es ein Dialog mit Vertreterinnen und Vertretern aller staatlichen Ebenen, der Wirtschaft, den Sozialpartnern, der Wissenschaft und der Zivilgesellschaft zur Verabredung von Aktivitäten. Konkret wurde der Dialog entlang der Themen in Arbeitsgruppen mit den relevanten Gestaltungspartnern organisiert:

- „Gute Partnerschaften für starke Familien"

- "Jugend gestaltet Zukunft"

- "Motiviert, qualifiziert und gesund arbeiten"

- „Selbstbestimmtes Leben im Alter"

- „Allianz für Menschen mit Demenz"

- „Regionen im demografischen Wandel stärken - Lebensqualität in Stadt und Land fördern"

- „Mobilisierung aller Potenziale zur Sicherung der Fachkräftebasis"

- „Ausländisches Arbeitskräftepotenzial erschließen und eine Willkommenskultur entwickeln"

- „Bildungsbiografien fördern"

- „Der öffentliche Dienst als attraktiver und moderner Arbeitgeber"

Die Arbeitsgruppe „Motiviert, qualifiziert und gesund arbeiten“ unter der Federführung des Bundesministeriums für Arbeit und Soziales sah in der Beratung von Unternehmen einen entscheidenden Ansatz. Mit dem Partnernetzwerk „Die Demografieexperten - DEx“ der Initiative Neue Qualität der Arbeit bestand bereits ein Beraternetzwerk mit Schwerpunkt auf dem demografischen Wandel. Es verabredeten sich die Kammern und vier Sozialversicherungsträger, in regionalen Modellvorhaben eine intensivere $\mathrm{Zu}$ sammenarbeit ihrer Berater zu organisieren, um besser das Wissen der anderen Organisationen in konkreten Fragestelllungen zum demografischen Wandel verfügbar zu machen. Das Bundesministerium für Arbeit und Sozialordnung startete zudem einen weiteren Ansatz, die Beratung zu forcieren, mit dem ESF-Programm „unternehmenswert: Mensch“2 die Beratung von kleinen und mittelständischen Unternehmen finanziell zu unterstützen. Die Aktivitäten zum Thema „Fachkräfte“ gingen auf in die Fachkräftestrategie der Bundesregierung. 


\section{Europäisches Jahr des aktiven Alterns ${ }^{3}$}

2012 war das Europäische Jahr des aktiven Alterns und der Solidarität zwischen den Generationen. Die Europäische Innovationspartnerschaft für Aktivität und Gesundheit im Alter hat Innovationen gefördert, um die Zahl der zu erwartenden gesunden Lebensjahre zu erhöhen. Ein Schwerpunkt des Europäischen Jahres 2012 lag darauf, angemessene Altersvorstellungen zu identifizieren. Denn wie erfüllt man seine zweite Lebenshälfte erlebt, hängt neben den äußeren Bedingungen auch von der eigenen Betrachtungsweise $a b$. In Deutschland übernahm das Bundesministerium für Familie, Senioren, Frauen und Jugend (BMFSFJ) die Umsetzung des Europäischen Jahres 2012. Organisatorisch unterstützt wird das BMFSFJ dabei von einer Geschäftsstelle. Diese wurde von der Bundesarbeitsgemeinschaft der Senioren-Organisationen e.V. (BAGSO) und der Forschungsgesellschaft für Gerontologie e.V. (FfG) getragen.

\section{Initiative Neue Qualität der Arbeit}

In der Arbeitswelt stellte sich konkret die Frage, wie mit älteren Belegschaften die Wettbewerbsfähigkeit erhalten werden kann. Und diese Frage stellte sich auch konkret für jede Mitarbeiterin, jeden Mitarbeiter persönlich. Die Arbeitsgestalter entwarfen beispielhaft Arbeitsplätze, die auf Beschäftigte im höheren Alter zugeschnitten waren. Die Gesundheit - der Erhalt der Arbeitsfähigkeit und die Stärkung der Beschäftigungsfähigkeit standen im Zentrum der Aktivitäten. Die vom BMAS ins Leben gerufene Initiative Neue Qualität der Arbeit stellte ihre Aktivitäten unter das Motto „30, 40, 50plus - gesund arbeiten bis ins Alter“. Um die Arbeitsfähigkeit zu erhalten, müssen die Arbeitsbedingungen zu den individuellen Fähigkeiten und Bedürfnissen passen - und zwar für jedes Alter ${ }^{4}$. Eine Reihe von Büchern, Broschüren, Leitfäden und Instrumenten wurden publiziert; andere Hilfsmittel wurden erstellt. Im Hinblick auf die Analyse wird auf die Altersstrukturanalyse, die Qualifikationsbedarfsanalyse sowie die alter $(\mathrm{n})$ kritische Gefährdungsbeurteilung verwiesen. Das Instrumentensetting reflektiert z.B. über Gesundheit, Arbeitsgestaltung, Führungs- und Unternehmenskultur, Personalplanung, -entwicklung oder -rekrutierung. Waren Instrumente oder Informationen nicht vorhanden, wurden sie im Rahmen der INQA-Projektförderung entwickelt, z.B.: 
- Strategische Personalplanung leicht gemacht - ein Handbuch sowie eine Hilfe für Betriebsräte für kleine und mittelgroße Unternehmen ${ }^{5}$

- Teams und Belegschaften systematisch entwickeln ${ }^{6}$

- Alle in eine Schublade - Altersstereotype erkennen und überwinden ${ }^{7}$

Eine alter(n)skritische Gefährdungsbeurteilung auf der Basis eines Arbeitsplatzkatasters wurde mit dem Projekt „ERGO-Stahl“8 erprobt, die auch die Sozialpartner bei der Umsetzung des Demografievertrages Stahl unterstützte. Das gleiche Ziel verfolgte z.B. das Projekt „Umsetzung von Demografieverträgen - DemTV“ in der Chemieindustrie. Mit dem Projekt PI$\mathrm{NA}^{9}$ - Gesund und qualifiziert älter werden in der Automobilindustrie“ wurden z.B. eine Kennzahlenlandkarte, ein Leitfaden für Führungskräfte oder eine Orientierungshilfe zur Arbeitsgestaltung entwickelt.

Nur „Wissen“ zur Verfügung zu stellen, reicht nicht aus. Es gründeten sich Partnernetzwerke der Initiative, die sich den Austausch, die Beratung oder die Anwendung von Instrumenten auf die Agenda gesetzt haben:

- Das Demographie Netzwerk e.V. ${ }^{10}$

- Die Demografie Experten - DEx ${ }^{11}$ oder

- Das WAI-Netzwerk ${ }^{12}$ und

- Der Initiativkreis „30, 40, 50plus - älter werden in Beschäftigung“

Das BMBF veranstaltete öffentlichkeitswirksam 2013 das Wissenschaftsjahr „Die demografische Chance.“

\section{Monitoring}

Die Bundesregierung hat den gesetzgebenden Körperschaften vom Jahr 2010 an alle vier Jahre über die Entwicklung der Beschäftigung älterer Arbeitnehmerinnen und Arbeitnehmer zu berichten und eine Einschätzung darüber abzugeben, ob die Anhebung der Regelaltersgrenze unter Berücksichtigung der Entwicklung der Arbeitsmarktlage sowie der wirtschaftlichen und sozialen Situation älterer Arbeitnehmerinnen und Arbeitnehmer weiterhin vertretbar erscheint und die getroffenen gesetzlichen Regelungen bestehen bleiben können. Der erste Bericht mit dem Titel Aufbruch in die arbeitsgerechte Arbeitswelt ${ }^{13}$ erfüllte nicht nur den gesetzlichen Auftrag. Ausführlich wurden Maßnahmen aufgezeigt, die in Unternehmen und Verwaltungen ergriffen wurden. Diesem Ansatz folgten auch die weiteren Fortschrittsberichte, so dass der dargestellte Anstieg der Beschäftigung Älterer auch mit einem Bild der Situation in Unternehmen und Verwaltungen korrespondiert. 


\section{Welche Fortschritte sind erzielt worden - welche Aufgaben bleiben?}

Erfreulich ist, dass die Beschäftigungsquote Älterer stark gestiegen ist. Die Statistik der Agentur für Arbeit gibt im September 2019 einen zusammenfassenden Bericht über die Situation von Älteren heraus. Die Autoren stellen fest, dass die Erwerbsneigung Älterer in den letzten Jahren im Vergleich zur Erwerbsneigung insgesamt überproportional stark zugenommen hat. Die Erwerbsquote stieg bei den Personen im Alter von 55 bis unter 60 Jahren von 2008 auf 2018 um 7,8 Prozentpunkte auf 83,0 Prozent. Einen sehr deutlichen Zuwachs gab es in der Gruppe der 60- bis unter 65Jährigen: Deren Erwerbsquote ist seit 2008 um 24,7 Prozentpunkte auf 62,5 Prozent gestiegen. Die gestiegene Erwerbsneigung Älterer betrifft beide Geschlechter. Zudem sind immer mehr Menschen auch über das Erreichen der Regelaltersgrenze hinaus erwerbstätig. Die Erwerbsneigung Älterer ist in Deutschland mit 73,6 Prozent im Jahr 2018 deutlich höher als im EU-Durchschnitt (EU-28: 62,0 Prozent). Das nationale EU-2020-Ziel, in Deutschland bis 2020 eine Erwerbstätigenquote für 55- bis unter 65-Jährige von 60 Prozent zu erreichen, wurde bereits 2012 übertroffen.

In der ersten Dekade des Jahrhunderts war der Blick auf die demografischen Herausforderungen fokussiert. Es zeigte sich, dass es als Querschnittsthema behandelt werden muss. Allein der Blick auf das Themenund Instrumentensetting zeigt die Spannbreite denkbarer Ansätze und Handlungsoptionen. Zunehmend traten auch andere Akteure auf den Plan, die weitere Aspekte von Vielfalt auf die Agenda setzen. Damit wurden die zentralen Herausforderungen des demografischen Wandels heute im Kontext weiterer Aspekte von „Vielfalt“ eingebettet, zum Beispiel

- Gender

- interkulturelle Zusammenarbeit oder

- Inklusion

Dieser Entwicklung wurde auch im Rahmen der Initiative Neue Qualität der Arbeit Rechnung getragen: Demografie wurde ein Aspekt des Handlungsfeldes „Vielfalt“. Das INQA-Instrumentarium, z.B. das INQA Audit, orientierte sich nach den maßgeblichen Handlungsfeldern Führung, Vielfalt, Gesundheit und Kompetenz. Das ddn setzt auf den Perspektivwechsel 3D: Demografischer Wandel, Digitalisierung und Diversity.

Weitere Aspekte der damaligen Aktivitäten wurden in anderen Zusammenhängen aufgegriffen und weitergeführt, z.B. die Unterstützung durch Beratung oder die Fachkräftestrategie der Bundesregierung. Das BMAS hat zudem in jedem der ostdeutschen Bundesländer ein Zukunftszentrum er- 
richtet, weil demografische und digitale Veränderungsprozesse in Ostdeutschland besonders zu spüren sind. ${ }^{14}$

In der 18. Legislaturperiode hatte das BMAS einen Dialogprozess „Arbeiten $4.0^{\text {“15 }}$ gestartet. Dieser war im Kern ein fachlicher Dialog über die Zukunft der Arbeitsgesellschaft. Es ging dabei jedoch nicht nur um ein Arbeiten in den neuen Produktionswelten der Industrie 4.0. Ziel war es insbesondere, auf Basis des Leitbilds "Guter Arbeit" vorausschauend die sozialen Bedingungen und Spielregeln der künftigen Arbeitsgesellschaft zu thematisieren und mitzugestalten. Zudem zeigte der Dialogprozess, zusammengefasst im „Weissbuch Arbeiten 4.0“16, neue Perspektiven und Gestaltungschancen, aber auch Spannungsfelder der Arbeitswelt der Zukunft auf. Der Dialogprozess verdeutlichte auch, dass Unternehmen die Entwicklung ihrer Geschäftstätigkeit immer weniger linear fortschreiben können. Digitalisierung, veränderte Markt- oder Produktionsverhältnisse, sich schnell ändernde Liefer- und Geschäftsverflechtungen bewirken, dass Unternehmen sich iterativ oder experimentell auf Veränderungen einstellen. Ein Ergebnis des Dialogprozesses war, dass das BMAS „Betriebliche Lernund Experimentierräume für Arbeitsinnovationen“ im Rahmen der Initiative Neue Qualität der Arbeit startete. Die Plattform „www.experimentierraeume.de ${ }^{17}$ zeigt Unternehmen, die sich experimentierend auf den Weg in die Arbeitswelt von morgen begeben haben. Zahlreiche geförderte Experimentierräume sind zudem in der Entwicklung. Im Ergebnis der KIStrategie der Bundesregierung werden im Herbst 2020 zudem Experimentierräume gestartet, die die Einführung von KI - gemeinsam gestaltet von Unternehmen und Beschäftigten - zum Ziel haben.

\section{Ausblick}

Veränderungen hat es immer gegeben. Der Zeithorizont, Veränderungen zu gestalten, ist ein entscheidender Faktor. Und es bedarf auch des Mutes und Vertrauens, sich auf etwas Neues einzulassen. In dieser Hinsicht sind die persönlichen Einstellungen, die Werte oder die Motivation sehr unterschiedlich. Diese Aussage trifft auch für eine einzelne Person über die verschiedenen Lebensphasen zu. Greift das Konzept einer alter(n)sgerechte Gestaltung der Arbeit noch angesichts agiler Arbeitsformen? Die gute Nachricht auf dem Weg in die Arbeitswelt von morgen ist sicherlich, dass durch Digitalisierung körperlich schwere Arbeit verhindert oder erleichtert werden kann. Absehbar wird es jedoch Beschäftigung in Gewerbe und Dienstleistung geben, die körperlich anstrengend oder monoton ist. Es bedarf jedoch größerer Anstrengungen, als die Beschäftigten in die moderne 
Arbeitswelt „mitzunehmen“. Es ist zu wenig und passt nicht in eine digitale Welt mit agilen Arbeitsstrukturen. Der Nutzen und Sinn modernen Arbeitens, z.B. Vorteile eines Arbeitens mit weniger Hierarchie erlebbar zu machen, ist erfolgsversprechend. So können Freiräume für Beschäftigte entstehen, in allen Lebensphasen ihre Arbeit mitzugestalten, so dass wir auch in Zukunft einen Anstieg der Beschäftigung in den älteren Kohorten verzeichnen können. Denn es ist nicht nur die Gesundheit. Motivation und soziale Dimensionen sind zentrale Faktoren für die Beschäftigung Älterer. Denn auch in Zukunft wird nach Ansicht von Prof. Juhani Ilmarinen das „Wollen, Können und Dürfen“ entscheidend für die Weiterbeschäftigung Älterer sein.

\section{Referenzen}

1 https://www.bundesregierung.de/breg-de/suche/eine-zusammenfassung-449622.

2 https://www.unternehmens-wert-mensch.de/startseite/.

3 https://ec.europa.eu/social/main.jsp?catId=1062\&langId=de.

4 https://www.inqa.de/DE/wissen/vielfalt/diversitaetsmanagement/demografischerwandel.html.

5 https://www.inqa.de/SharedDocs/downloads/strategische-personalplanung-leichtgemacht.pdf?_blob=publicationFile\&v=6, https://www.inqa.de/SharedDocs/dow nloads/strategische-personalplanung-mit-weitblick.pdf?_blob=publicationFile\&v $=3$.

6 https://www.inqa.de/SharedDocs/downloads/teams-und-belegschaften-systematisc h-entwickeln.pdf;jsessionid=C4C1AEFF1E585021326B18CCE65600DD.delivery2 -replication?_blob=publicationFile $\& v=2$.

7 https://www.inqa.de/SharedDocs/downloads/alle-in-eine-schublade-altersstereoty pe-erkennen-und-ueberwinden.pdf?_blob=publicationFile\&v $=2$.

8 https://www.bit-bochum.de/leistungen/forschung/archiv/ergo-stahl/.

9 https://pina-projekt.de/.

10 https://demographie-netzwerk.de/.

11 http://www.demografie-experten.de/.

12 https://www.wainetzwerk.de/de/.

13 http://www.bmas.de/SharedDocs/Downloads/DE/PDF-Publikationen/anlage-beri cht-der-bundesregierung-anhebung-regelaltersgrenze.pdf?_blob=publicationFile.

14 https:/www.bmas.de/DE/Presse/Pressemitteilungen/2019/ostdeutsche-bundeslae nder-zukunftszentren.html.

15 https://www.bmas.de/DE/Themen/Arbeitsmarkt/Arbeiten-vier-null/arbeiten-viernull.html.

16 https://www.bmas.de/DE/Service/Medien/Publikationen/a883-weissbuch.html.

17 https://www.experimentierraeume.de/start/ wird alsbald unter INQA.de zu finden sein. 
